\title{
La gouvernance de la conciliation travail-famille : comparaison entre la France et le Québec
}

Marie-Agnès Barrère-Maurisson - FRANCE

Chercheuse au Centre national de recherche scientifique, Centre d'économie de la Sorbonne

Diane-Gabrielle Tremblay - ouÉBEC

Professeure titulaire, Université du Québec à Montréal (Télé-université)

\section{RÉSUMÉ} tion travail-famille, il est rare de mettre en présence tous les acteurs des politiques. Nous avons conçu un programme qui vise non seulement à dresser un état des lieux comparatif basé sur les données démographiques et d'emploi produites tant en France qu'au Québec, mais aussi à faire se rencontrer les acteurs intermédiaires que sont les entreprises, les organisations syndicales, les administrations régionales ou municipales, les institutions en charge de l'enfance et de la famille ainsi que les associations d'économie sociale. À partir d'une revue des écrits, d'échanges de propos en visioconférences et d'un séminaire annuel ouvert aux gouvernants, nous avons commencé à confronter les pratiques utilisées de part et d'autre, selon trois objectifs : éclairer les décideurs politiques et les autres acteurs; mieux connaître les enjeux de la question ainsi que les limites ou les effets pervers de certaines actions; déceler les pistes et les leviers possibles. Ces travaux permettront de faire le lien entre le gouvernement et la gouvernance en matière de conciliation travail-famille. Le présent article fait état des premières observations sur le plan statistique comme en ce qui concerne le rôle des acteurs intermédiaires dans les deux sociétés.
ABSTRACT On the highly topical economic and social issue of work-family balance, it is unusual that all policy actors are brought together. We have designed a program that not only draws up a comparative assessment of the situation based on the demographic and employment data produced in France and Québec, but also brings together the intermediary actors as represented by companies, union organizations, regional or municipal administrations, institutions in charge of childhood and family issues, and the social economy associations. Based on a review of the literature, of discussions via videoconferences and of $a$ yearly seminar open to managers, the practices used by each party were compared, in light of three goals: to inform policymakers and other actors, to gain a better understanding of the challenges involved in the issue and the limits or perverse effects of some actions, and to identify the potential courses of action and levers. This endeavour will help to link the government with management regarding work-family balance. This article reports on the first statistical observations about the role of intermediary actors in both societies. 
$\mathrm{N}$ ombre de travaux ont fait état d'un lien entre fécondité et activité féminine (Barrère-Maurisson, 2006; Cette et al., 2007). Ce constat nous a amenées à vouloir comparer non seulement les situations de la France et du Québec, mais aussi les rôles que peuvent jouer certains acteurs et certaines politiques. Bien que ces deux sociétés soient comparables du point de vue de la conciliation travail-famille, au regard du taux d'activité notamment, on y trouve certaines différences sur les plans statistique et institutionnel, ce qui rend la comparaison intéressante. En effet, au-delà des ressemblances, on peut remarquer des différences institutionnelles ou de politiques qui méritent un approfondissement. De plus, avec le redéploiement de l'État-providence, divers acteurs reviennent sur le devant de la scène; il s'agit d'acteurs que nous avons qualifiés d'intermédiaires, du fait de leur positionnement entre les décideurs et les bénéficiaires des mesures, programmes ou politiques. On peut penser ici aux associations patronales et syndicales, à des associations s'intéressant aux questions de famille et de conciliation sous divers angles ou, encore, aux administrations régionales et municipales, qui investissent ce nouveau champ. Si les syndicats et les associations peuvent représenter les bénéficiaires, les entreprises et les collectivités locales peuvent quant à elles contribuer à la mise en œuvre de solutions à l'intention de ces mêmes bénéficiaires, de sorte que nous les regroupons sous le terme d'acteurs intermédiaires.

C'est dans ce contexte que nous avons entrepris la comparaison statistique, en nous interrogeant sur les sources des ressemblances et des différences, et plus particulièrement sur le rôle des acteurs intermédiaires. Ainsi, nous avons lancé le programme de recherche Conciliation travail-famille: état des lieux et pratiques des acteurs «intermédiaires»comparaisons France-Québec. Par ce programme, nous étudions les pratiques de ces divers acteurs afin de déterminer comment la place et le rôle de chacun contribuent à configurer des modèles de gouvernance spécifiques. Le projet se poursuivra jusqu'à la fin de 2009, mais un certain nombre de résultats peuvent déjà être exposés.

Dans le présent article, nous précisons d'abord le cadrage statistique, puis nous faisons état de la réflexion concernant les modèles de gouvernance, à partir des recherches et des échanges avec les acteurs intermédiaires menés au cours de la dernière année.

\section{Le contexte de la conciliation travail-famille en France et au Québec}

Les difficultés de la conciliation entre vie professionnelle et vie familiale incitent de nombreuses personnes - essentiellement des femmes, en raison de la division sexuelle du travail - à renoncer soit à un enfant supplémentaire ou, durant certaines périodes de leur vie et de celle de leurs enfants, à un fort investissement professionnel, voire à un emploi (Barrère-Maurisson, 2003; Cette et al., 2007).

\section{Un lien entre fécondité et activité féminine}

Dans certains pays de l'Europe du Sud, dont l'Espagne, la Grèce et l'Italie, la difficile conciliation travail-famille contribue à de faibles taux de fécondité et à de faibles taux d'activité des femmes. Par contre, d'autres pays - la Finlande et la Suède, notamment connaissent à la fois des taux de fécondité et des taux d'activité des femmes relativement élevés (Cette et al., 2007).

Pour sa part, la France constitue à l'heure actuelle une «exception», alliant l'un des plus forts taux de fécondité (1,94 en 2006, ce qui la place au $2^{\mathrm{e}}$ rang européen après l'Irlande) et un fort taux d'activité féminine (plus de $80 \%$ des mères de un ou deux enfants sont actives) selon l'enquête Emploi 2005 de l'INSEE (2008). Qui plus est, à la différence d'autres Européennes, les Françaises sont majoritairement actives à temps plein et de façon continue. C'est donc la conjugaison de ces deux facteurs, fécondité et activité, qui fonde aujourd'hui «l'exception française» (BarrèreMaurisson, 2006).

Il est intéressant d'approfondir l'analyse des taux d'activité et la répartition des temps sociaux en France et au Québec, d'autant plus que le Québec vient d'adopter une nouvelle politique en matière de congé parental en 2006 (Tremblay, 2008a) et que la France 
a établi le congé de paternité en 2002 après d'autres mesures telles que l'Allocation parentale d'éducation (APE) ou la Prestation d'accueil du jeune enfant (PAJE). Les diverses mesures instaurées de part et d'autre peuvent influer non seulement sur les taux d'activité ou les taux de fécondité, mais aussi sur l'égalité en emploi et le maintien en poste de la main-d'œuvre. Tout cela prend de l'importance dans un contexte où l'équité en emploi est mise en avant dans les deux sociétés et où celles-ci doivent composer avec le vieillissement de la main-d'œuvre. Cette dernière réalité touche le Québec plus intensément que le reste de l'Amérique du Nord mais autant que la France, où le vieillissement de la population a commencé plus tôt et les retraits du marché du travail sont plus précoces qu'au Québec. Soulignons que le nouveau congé parental mis en place au Québec semble avoir contribué à la hausse du taux de fécondité, lequel est passé de 1,5 à 1,6 depuis 2006 (Tremblay, 2008c).

Nombre de travaux montrent que des difficultés à concilier les activités professionnelles avec la vie personnelle et la vie familiale sont à l'origine, en grande partie, des inégalités entre hommes et femmes sur le marché du travail, de la faible fécondité, de la réduction de l'activité sur le marché du travail et des problèmes à maintenir en poste tant la main- d'œuvre féminine que la main-d'œuvre vieillissante en général (Barrère-Maurisson, 2004; Tremblay, 2008b).

Outre l'importance de la question tant du point de vue social que du point de vue de l'équité en emploi des hommes et des femmes, des avantages sur le plan économique pourraient être attendus de politiques visant à faciliter cette conciliation: d'une part, la hausse du taux d'activité des femmes contribuerait à élever le niveau potentiel du PIB; d'autre part, l'augmentation de la fécondité accélérerait la croissance et, de ce fait, le niveau de vie de la population s'améliorerait (Cette et al., 2007), laquelle amélioration favorierait éventuellement l'équité entre hommes et femmes - celle-ci étant plus facile à atteindre lorsque l'économie est en progression, même faiblement.

\section{France-Québec : \\ des ressemblances mais aussi des différences}

Soulignons d'abord que la participation des femmes au marché du travail n’a cessé de croître depuis les années 70 (figure 1), mais une analyse plus approfondie met en évidence des écarts non négligeables selon le nombre d'enfants et l'âge de ces derniers (tableau 1). Dans le cas des mères ayant au

F I G U R E 1 Taux d'activité sur le marché du travail des femmes de 15 à 64 ans (\%)

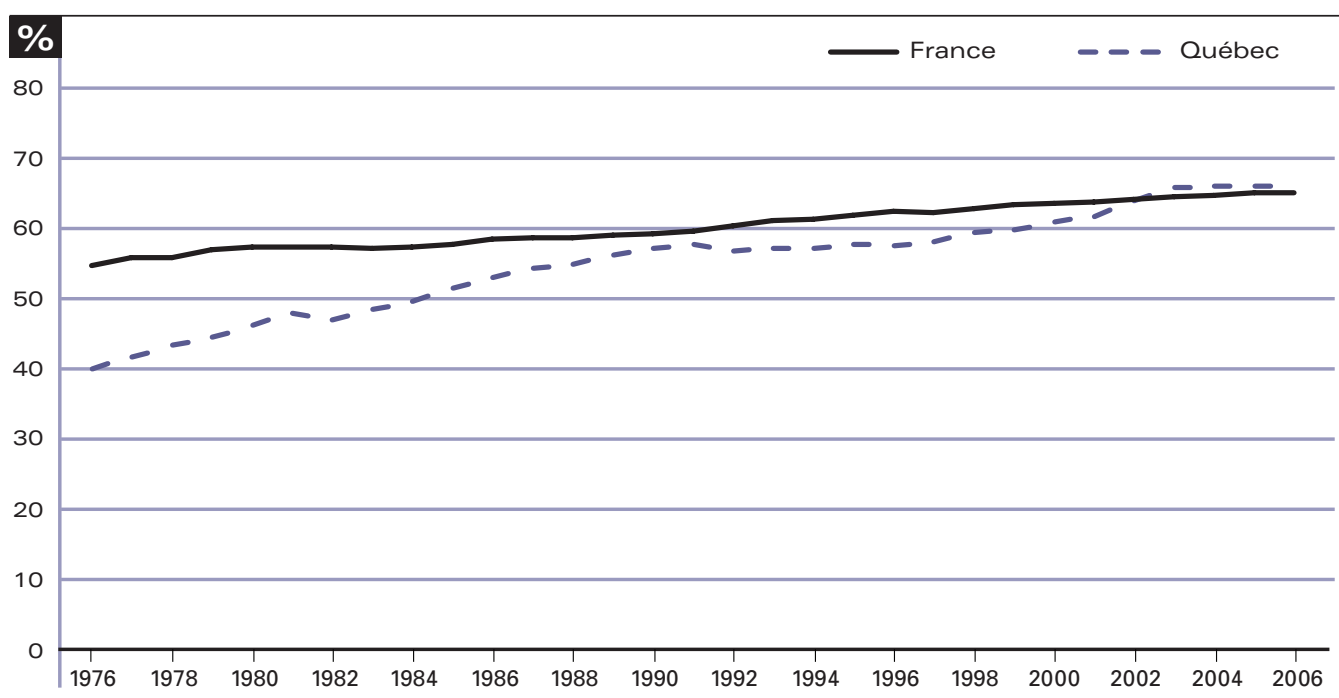

Sources: France: INSEE, INSEE Résultats "Séries longues sur le marché du travail», enquêtes Emploi $1975-2006$. Québec: Statistique Canada, Enquête sur la population active. 
T A B E A U 1 Taux d'activité sur le marché du travail des parents ayant au moins un enfant, selon l'âge du plus jeune (2003) (\%)

\section{ÂGE DU PLUS JEUNE ENFANT}

Moins de 3 ans

De 3 ans à moins de 6 ans

De 6 ans à moins de 18 ans

18 ans ou plus

Hommes
\begin{tabular}{|l|l|l|l|l|l} 
France & \multicolumn{3}{l}{ Femmes } & Ensemble \\
\hline 60,2 & 73,3 & 96,4 & 94,1 & 78,2 & 83,7 \\
74,4 & 77,2 & 96,7 & 95,0 & 84,9 & 86,1 \\
82,8 & 83,7 & 96,4 & 95,2 & 88,8 & 89,5 \\
83,0 & n. d. & 92,7 & n. d. & 87,8 & n. d.
\end{tabular}

Sources: France: Cette et al. (2007) à partir de l'enquête Emploi 2003; INSEE, enquête Emploi 2005. Québec: Statistique Canada, Enquête sur la population active.

moins un enfant âgé de moins de 3 ans, le taux d'activité des Québécoises était de 73,3\%, contre 60,2\% pour les Françaises, en 2003. D'autres statistiques montrent que le taux d'activité des Françaises dans ce cas est très sensible au nombre d'enfants, sans doute en raison des prestations PAJE et APE, qui encouragent les parents de deux enfants ou plus à quitter temporairement le marché du travail. Ainsi, le taux d'activité des Françaises dont le plus jeune enfant est âgé de moins de 3 ans chute à $37,5 \%$ lorsqu'elles ont trois enfants ou plus, alors qu'il s'établit respectivement à $59,8 \%$ pour les mères de deux enfants et à $81,3 \%$ pour les mères d'un enfant (INSEE, 2008).

Malgré l'augmentation constante des taux d'activité des femmes, de fortes inégalités subsistent entre hommes et femmes. Des deux côtés de l'Atlantique, les femmes sont plus nombreuses à occuper des emplois précaires ou à temps partiel (Lavallée, 2002; Lemière, Marc, 2006), l'emploi féminin est fortement concentré dans certains secteurs tels que la santé, l'éducation ou les services sociaux et les femmes sont toujours sous-représentées dans les postes à responsabilités, que ce soit dans le secteur public ou privé (Colin et al., 2005; Tremblay, 2004). Enfin, les différences de rémunération persistent en dépit des politiques favorisant la parité (ISQ, 2008; Petit, 2006).

Les Françaises et les Québécoises sont toujours en première ligne pour accomplir les tâches domestiques et pour s'occuper des enfants ainsi que des personnes âgées (tableau 2). En France, en 1998, les femmes assuraient encore près de $70 \%$ du travail domestique (Anxo, 2003; Barrère-Maurisson et al., 2000); la situation semble un peu plus égalitaire au Québec, où les femmes en assuraient $63 \%$ en 1998 et $57 \%$ en 2005 (Statistique Canada, 2006). Les femmes ont toutefois moins de temps libre que les hommes. En résumé, la parité a progressé mais des inégalités persistent, en particulier au regard du temps.

Dans l'ensemble, les femmes consacrent au travail non rémunéré une part de leur temps qui représente près du double de celle qu'y consacrent les hommes, au Québec comme en France (Barrère-Maurisson, 2004; Statistique Canada, 2006). Elles allouent même plus de temps aux activités parentales et domestiques qu’au travail rémunéré (Anxo, 2003; Statistique Canada, 2006).

Ainsi, en dépit de grandes similitudes dans la façon dont les ménages français et québécois emploient leur temps, on constate une répartition plus égalitaire au Québec qu'en France. Les Québécoises participent davantage au marché de l'emploi que les Françaises (Cette et al., 2007) et les Québécois assument une part plus importante du travail domestique que les Français (Tremblay, 2003 et 2008a).

Cela étant, le statut d'emploi, tant des femmes que des hommes, est une variable cruciale pour prédire la répartition des tâches domestiques en France et au Québec (Anxo et al., 2001; Tremblay, 2003 et 2008a). Les écarts de salaire entre les conjoints accentuent les inégalités au regard de la division des 
T A B L E A U 2 Part du temps consacré par les femmes par rapport au temps total consacré par le couple, selon l'activité (\%)

France (1998)

Québec (1998)

Québec (2005)

Tâches professionnelles
\begin{tabular}{|l|l|} 
& Tâches domestiques \\
36 & 68 \\
43 & 63 \\
46 & 57
\end{tabular}

Dont soins aux tiers ${ }^{1} \quad$ Temps libre

1. Inclus dans les tâches domestiques.

Champ: France: ensemble des personnes actives ou non, âgées de 18 à 64 ans et vivant en couple ; Québec 1998: population de 15 ans et plus vivant en couple; Québec 2005: population active de 15 ans et plus.

Lecture: France: $36 \%$ du temps total consacré aux tâches professionnelles dans le couple sont effectuées par les femmes (64\% par les hommes).

Sources: France: INSEE, Enquête Emploi du temps.

Québec: Statistique Canada, Enquête sociale générale 1998 et 2005.

tâches au sein du couple. Dans les couples à deux actifs non équivalents - homme travaillant à temps complet et femme travaillant à temps partiel par exemple, profil en forte progression en France jusqu'à la fin des années 90 -, on constate une suraccumulation des temps professionnel et familial pour les conjointes (Barrère-Maurisson, 2004). Ainsi, le statut d'emploi de la femme est un facteur plus important que celui de l'homme pour expliquer la répartition plus ou moins équitable des tâches domestiques. Lorsque les femmes occupent un poste à temps plein, le partage des tâches tend à être plus équitable. La répartition des tâches au sein des couples reste encore éloignée de la parité, mais la structure de la famille et de l'emploi des conjoints permet d'expliquer des répartitions plus ou moins inégalitaires. La répartition du travail domestique est proportionnelle à l'investissement professionnel de chacun. Ainsi, lorsque l'un des deux conjoints est au foyer, il assume totalement les charges domestiques et familiales. En revanche, lorsque les deux membres du couple appartiennent aux mêmes catégories socioprofessionnelles et qu'ils ont des revenus identiques, la répartition est plus équilibrée (Bauer, 2007). En France comme au Québec, plus les écarts de revenu entre les conjoints sont importants, plus la spécialisation des rôles reste forte.

La prise en charge des jeunes enfants a aussi un effet important sur l'activité. Cette question se pose, en France, surtout de 0 à 3 ans, en raison de la scolarisation importante des enfants de 3 à 6 ans, mais aussi de l'insuffisance des services de garde de 0 à 3 ans. Au Québec, les enfants entrent à l'école maternelle à 5 ans; la garde de la petite enfance se pose donc généralement de 0 à 5 ans mais, à partir de 6 mois, les enfants peuvent être accueillis dans les centres de la petite enfance. Beaucoup le sont à partir de 1 an, le congé parental étant d'environ 12 mois et pouvant être partagé entre le père et la mère (Tremblay, 2008 a et $2008 b$ ).

\section{Deux modèles de gouvernance: partenaires versus acteurs}

Avec le redéploiement des régimes d'Étatprovidence, les entreprises et les collectivités territoriales, entre autres, reprennent un rôle dans la gestion des problèmes économiques et sociaux nationaux (Barrère-Maurisson, 2003). Du fait de la décentralisation plus importante des politiques, il est intéressant d'analyser, de façon à faire une comparaison entre les deux sociétés, le rôle de ces acteurs que nous qualifions d'intermédiaires.

\section{Des formes de gouvernance face à des gouvernements}

Le mot anglais governance est, depuis les années 90, utilisé sous la forme française gouvernance dans le sens d'«art ou manière de gouverner». Ce mot marque la distinction avec le gouvernement et sert à promouvoir 
un nouveau mode de gestion des affaires publiques fondé sur la participation de la société civile à tous les niveaux: local, régional, national et international.

La gouvernance correspond à une forme post-moderne des organisations, utilisant ce nouveau mode de gestion publique, et renvoie à une régulation sociale maintenant plus complexe. Du fait de la régression de la prise en charge nationale, de l'engagement grandissant de l'échelon supranational et du réengagement des acteurs intermédiaires, nous avons qualifié ce nouveau mode de régulation sociale de multipartite (Barrère-Maurisson, 1995 et 2003).

En ce sens, il convient ici de mettre en lumière la production de la gouvernance en matière de conciliation travail-famille ${ }^{1}$, de façon à pouvoir, le cas échéant, par confrontation avec les options nationales, mettre en relation la gouvernance locale en la matière, soit les acteurs intermédiaires, avec son gouvernement, soit les organismes gouvernementaux.

\section{Modèles de société et politiques favorisant la conciliation}

L'histoire récente des politiques et des institutions permet de comprendre comment s'opère la régulation de la conciliation travailfamille de chaque côté de l'Atlantique. Au cours des cinquante dernières années, la France est progressivement passée d'une régulation fondée sur la préservation de la famille - le familialisme -, à une régulation cherchant à préserver la place des femmes le féminisme -; aujourd'hui, c'est la préoccupation sociale de l'enfant et de la parentalité qui prédomine, d'où la dénomination de parentalisme (Barrère-Maurisson, 2007).

Les années 60 et 70 ont été marquées par une importante salarisation de la main-d'œuvre féminine, conjointement au développement du secteur tertiaire. De nouvelles formes de la famille sont apparues: on est passé d'une famille de type conjugal, où seul le chef de famille travaillait, à une famille où homme et femme étaient actifs, même si la femme ne l'était pas autant que le mari. C'est alors la fin du modèle familialiste, dans lequel la famille assurait une sorte d'autorégulation et constituait un objet prioritaire des politiques. Le même type d'évolution a été observé au Québec.

Les années 80 sont marquées, en France comme au Québec, par un marché du travail plus flexible. Le travail des femmes progresse encore et contribue, au cours de la décennie suivante, à fixer un nouveau type de famille: les familles à deux actifs équivalents. C'est l'âge du féminisme en tant que mode de régulation sociale. Les affaires de la famille sont encore majoritairement une affaire de femmes; celles-ci, objet central des politiques publiques, continuent d'assurer la régulation entre la sphère privée et la sphère professionnelle ${ }^{2}$.

Depuis les années 90, la flexibilité et la précarité deviennent structurelles avec la généralisation du contrat à durée déterminée dans le milieu du travail et une plus grande instabilité des familles et des couples. L'objet des politiques et du droit de la famille devient l'enfant, seul repère désormais par rapport à une structure qui a perdu son cadre stable et durable. On peut y voir un nouvel ordre social, que nous avons appelé le parentalisme. Celuici tend en effet à se développer à l'échelle de la société par la mise en place progressive et de façon convergente de mesures centrées autour du renforcement de la parentalité dans différents domaines tels que l'emploi et l'entreprise, notamment ${ }^{3}$.

Le bref historique qui précède éclaire la situation démographique exceptionnelle de la France d'aujourd'hui. Rappelons que l'«exception française» tient, à notre sens, à la conjonction d'un taux de fécondité élevé et d'un taux d'activité féminine important.

Cette situation s'explique par le fait que la France, à la différence des autres pays européens et de ceux de l'Amérique du Nord, a toujours défini, depuis 1945, une politique démographique - la population étant considérée comme une force politique et économique. La mise en place progressive de mesures publiques de conciliation telles que

1. Voir Eme et Fraisse (2005) sur la gouvernance locale à propos de la régulation des services de la petite enfance.

2. On parle aussi de "féminisme d'État" (Revillard, 2006).

3. Pour le Canada, Jenson (2004) évoque un changement de paradigme vers un partage des responsabilités entre parents et collectivité, tandis que Dandurand (2005) parle de "parentage multiple" à propos de la participation croissante des instances publiques auprès des enfants et des parents. 
l'APE, la PAJE-CLCA (complément de libre choix d'activitét) ou le congé de paternité a permis une articulation nouvelle entre politiques familiales et politiques sur l'emploi. Au Québec, la transition démographique s'est opérée plus tardivement et de façon plus brutale, avec le passage d'une forte natalité à un déclin soudain, sans accompagnement politique. Cependant, ces dernières années, on observe, après la mise en place d'un congé parental allongé, mieux rémunéré et favorisant les pères, une progression du taux de fécondité (Tremblay, 2008a et 2008b).

Certes, la question des politiques familiales et de la conciliation travail-famille se présente différemment en France et au Québec, car elle correspond à un enjeu de société sensiblement distinct. En France, en particulier, il existe une politique familiale objectivée et la famille est une réelle question économique et sociale. Au Québec, ce sont les mouvements sociaux qui ont porté le débat depuis trois décennies. Si l'on se réfère à une typologie des sociétés ${ }^{5}$, on peut citer les trois modèles suivants: le premier, anglo-saxon, que l'on retrouve aux États-Unis et au Canada anglais, est de type libéral, où la famille est une question privée; le second, franco-nordique, est de type étatique, la famille étant une affaire d'État et la politique démographique relevant du gouvernement; le troisième modèle, auquel répond le Québec, fait une large part à la gouvernance en matière de famille, les acteurs sociaux - dont les entreprises, les syndicats et les associations - ayant participé très activement à la revendication puis à la mise en place de mesures telles que le congé parental et les centres de la petite enfance (Conseil de la famille et de l'enfance, 2007).

\section{Les modalités de la gouvernance au regard de la conciliation travail-famille}

En France comme au Québec, la scène publique s'est enrichie de la participation des différents acteurs intermédiaires tels que les collectivités locales, les associations, les syndicats et les entreprises.

\section{De nouveaux acteurs dans \\ I'intervention publique}

C'est dorénavant par les acteurs intermédiaires, notamment, que se mettent en place les conditions assurant une meilleure articulation entre la vie professionnelle et la vie familiale. Ainsi, certaines entreprises ont adopté des politiques sur la conciliation travail-famille par l'intermédiaire des programmes favorisant l'égalité. La France a instauré le label Égalité et, récemment, elle a établi des procédures liées à la protection de la parentalité dans l'emploi ${ }^{6}$. Les entreprises sont sensibles à ces préoccupations et en font, dans un contexte de possible pénurie de main-d'œuvre, un argument pour attirer et garder leurs salariés. L'État s'y intéresse également, trouvant là un relais soit à des politiques plus globales concernant l'aide à la famille ou à l'emploi, soit à des recommandations européennes liées à la stratégie de Lisbonne. Au Québec, le prix ISO-famille récompense les entreprises qui préconisent des «bonnes pratiques»; l'influence du gouvernement canadien, plus conservateur et moins actif sur le plan de la conciliation, se fait cependant sentir.

La convergence des objectifs illustre la place que prennent désormais les acteurs intermédiaires dans la gouvernance de la conciliation travail-famille. Ces acteurs ne sont pas nouveaux, mais ils assument des rôles inédits en la matière. En analysant les processus de coordination entre les divers échelons, on peut saisir en quoi on a affaire à des modèles de gouvernance distincts de part et d'autre de l'Atlantique.

On en arrive à penser que la gouvernance peut constituer un moyen de répondre à la crise de nos régimes d'État-providence par de nouvelles formes de régulation. La crise de la gouvernabilité incite en effet les États à se tourner vers de nouveaux interlocuteurs pour

4. La PAJE ne se résume pas au CLCA; si ce dernier tend à réduire la participation des femmes au marché du travail, le complément de libre choix du mode de garde (CMG), qui fait partie de la PAJE, soutient au contraire I'activité des parents.

5. Diverses typologies des sociétés ont été proposées. L'espace imparti ici ne permet pas de les détailler mais le lecteur qui veut en savoir plus peut consulter Tremblay (2008a) ou Barrère-Maurisson (2003).

6. Nous avons prôné, il y a peu, la mise en place en France du label Parentalité, sur le même mode que le label Égalité; le ministre du Travail et la secrétaire d'État à la Famille ont présenté, le 11 avril 2008, la Charte de la parentalité en entreprise, qui a pour objectif d'inciter les entreprises à proposer aux salariés-parents un environnement mieux adapté aux responsabilités familiales. 
proposer des solutions. Si des organismes intermédiaires tels que les syndicats et les associations peuvent représenter les bénéficiaires, d'autres - comme les entreprises et les collectivités locales - peuvent être appelés à participer à la mise en œuvre des solutions. Cela conduit à un changement du rôle des autorités publiques: d'interventionnistes, elles deviennent facilitatrices, animatrices ou régulatrices, suivant les cas.

\section{Se doter d'outils}

Dans le cadre de notre programme de recherche, cinq acteurs intermédiaires ont été identifiés: les entreprises, les organisations syndicales, les administrations régionales ou municipales, les institutions en charge de l'enfance et de la famille ainsi que les associations d'économie sociale. Nous analysons les pratiques et les rôles de chacun au regard de la conciliation travail-famille, de façon comparable dans les deux sociétés.

Nous nous intéressons aux pratiques et aux mesures des entreprises - particulièrement les grandes entreprises, qui ont les moyens de mettre en œuvre des mesures de conciliation - et à celles des organisations syndicales. En France et au Québec, force est de constater que l'objectif de la conciliation semble se diluer dans ceux de l'égalité professionnelle et de la diversité et que les entreprises n'ont pas encore vraiment compris l’importance des questions relatives à la conciliation, et ce malgré la récente Charte de la parentalité adoptée en France. Il apparaît donc nécessaire de concevoir des outils permettant de mesurer les progrès en la matière pour pouvoir ensuite créer une émulation.

Par ailleurs, la façon de présenter les politiques est apparue problématique, notamment par rapport aux administrations régionales et municipales. Doit-on promouvoir la conciliation par l'intermédiaire d'une politique sur les temps sociaux, à linstar des politiques de la ville, ou en la liant à l'égalité hommes-femmes? Doit-on cibler des populations et, dans l'affirmative, lesquelles: les femmes, les pères?

\section{Questions de coordination}

La notion de gouvernance permet de saisir l'interdépendance des pouvoirs, car la nouvelle gestion des affaires publiques repose sur un processus d'interaction et de négociation entre les acteurs de chaque niveau - local, régional et national. La question de la coordination se pose, de même que la nécessité de définir les rôles de chacun.

Si le Québec a une pratique relativement ancienne des initiatives locales, la France, elle, est sous le régime de la régionalisation seulement depuis les lois de 1982 et 1983; toutefois, elle se trouve assujettie à différentes régulations, y compris au niveau supranational. La multiplicité des règles et des normes constitue une préoccupation majeure pour les administrations régionales ou municipales, que partagent d'une autre façon les institutions en charge de l'enfance et de la famille. En France, l'offre relative à l'accueil de la petite enfance est très diversifiée et la question de sa régulation se pose a priori, du fait de l'intervention de multiples acteurs: les parents, les municipalités, les caisses d'allocations familiales, etc. Au Québec, l'offre est relativement unifiée, mais ce sont les acteurs - entreprises et associations, notamment - qui prennent des initiatives que le gouvernement vient ensuite soutenir ou encadrer, comme dans le cas des services de garde.

Ainsi, on peut se demander s'il n'y a pas là deux modèles distincts de gouvernance: le modèle français, basé sur la coordination des actions et sur des institutions qui sont des partenaires, et le modèle québécois, s'appuyant sur une gouvernance des initiatives prises par des acteurs qui se chargent de les mener à bien.

\section{Conclusion}

Afin de conjuguer tous les impératifs - de nature démographique (reprise de la fécondité dans nos sociétés vieillissantes), économique (compétitivité des économies), politique (égalité des sexes) ou sociale (prise en charge domestique et parentale) - auxquels nos sociétés doivent satisfaire, il est aujourd'hui nécessaire de se fixer des objectifs concrets, notamment en matière de conciliation travailfamille.

Pour cela, et au-delà d'objectifs globaux comme l'égalité - souvent seulement politique ou professionnelle - et la diversité, il faut amener la conciliation travail-famille au premier plan et en faire un objectif sociopolitique. De ce choix dépend, en effet, l'adaptation 
réciproque des structures productives et reproductives de nos sociétés post-modernes. C'est particulièrement le cas en ce qui concerne la prise en charge de la parentalité par les milieux de travail, l'évolution des rôles parentaux notamment celui des pères - et la transformation des types de famille - notamment au regard des modalités relatives à l'union et à la filiation (Barrère-Maurisson, 2004).

Dès que la conciliation travail-famille a valeur d'objectif sociopolitique, la nouvelle gouvernance de l'emploi ou du marché du travail - basée sur la flexicurité, notamment peut être envisagée, d'une certaine façon, comme le pendant de la gouvernance de la famille (Barrère-Maurisson, 2007, p. 25). En d'autres mots, face à la flexibilité des structures, on observe une recherche de la sécurisation du lien social, celle-ci passant par la sollicitation d'une multiplicité d'acteurs sociaux (Tremblay, 2008d).

Dans ce sens, le parentalisme, ou modèle de société régulée par des politiques convergeant vers une meilleure prise en charge de la parentalité - sous tous ses aspects -, est aujourd'hui l'une des voies permettant le développement de la conciliation travailfamille, grâce à la gestion conjointe de toutes les institutions: supranationales, gouvernementales et intermédiaires.

Peut-être est-ce à ce prix que pourra être assuré le devenir de nos sociétés développées, désormais multipartites, telles que le Québec et la France.

\section{Bibliographie}

Anxo D. (2003). Division sexuelle des tâches: Les expériences françaises et suédoises, Futuribles, no $285,33-40$.

Anxo D., Boulin J.Y., Lallement M., Lefevre G., Silvera R. (2001). Recomposition du temps de travail, rythmes sociaux et modes de vie: Une comparaison France-Suède, Travail et Emploi, $\mathrm{n}^{\mathrm{0}}$ 74, 5-20.

Barrère-Maurisson M.A. (2007). Familialisme, féminisme et «parentalisme»: trois âges de la régulation sociale, Cahiers de la Maison des sciences économiques, $\mathrm{n}^{\circ}$ 43, 29.

En ligne: <http:/halshs.archives-ouvertes.fr/halshs-00175883/fr >.

Barrère-Maurisson M.A. (2006). Les femmes françaises créent l'exception.

En ligne: <http://halshs.ccsd.cnrs.fr/halshs-00009702>.

Barrère-Maurisson M.A. (2004). Masculin/féminin : vers un nouveau partage des rôles?, Cahiers français, $\mathrm{n}^{\circ}$ 322, 22-28.

En ligne: <http://halshs.archives-ouvertes.fr/halshs-00115868>.

Barrère-Maurisson M.A. (2003). Travail, famille: le nouveau contrat, Paris, Gallimard, «Folio actuel - Le Monde», 210.

En ligne: <http://matisse.univ-paris1.fr/travail-famille et http://halshs.archives-ouvertes. fr/halshs-00010450>.

Barrère-Maurisson M.A. (1995). Régulation familiale, marchande ou politique : les variations de la relation travail-famille, Sociologie et Sociétés, 27 (2): 69-85.

En ligne: <http:/www.erudit.org/revue/socsoc/1995/v27/n2/001023ar.pdf>.

Barrère-Maurisson M.A., Rivier S., Marchand O. (2000). Temps de travail, temps parental La charge parentale: un travail à mi-temps, Premières Synthèses, no 20.1, 7.

En ligne: <http://www.travail-solidarite.gouv.fr/etudes-recherche-statistiques-dares/ etudes-recherche/publications-dares/premieres-informations-premieres-syntheses/200020.1-temps-travail-temps-parental-charge-parentale-travail-mi-temps.html>.

Bauer D. (2007). Entre maison, enfant(s) et travail: les diverses formes d'arrangement dans les couples, Études et Résultats, n ${ }^{\circ}$ 570, 8.

Cette G., Méda D., Sylvain A., Tremblay D.G. (2007). Activité d'emploi et difficultés de conciliation emploi-famille: une comparaison fine des taux d'activité en France et au Canada, Loisir et Société/Society and Leisure, 29 (1): 117-154. 
Colin C., Djider Z., Ravel C. (2005). La parité à pas comptés, INSEE Première, no 1006, 4.

Conseil de la famille et de l'enfance (2007, révisé 2008). La politique familiale au Québec: visée, portée, durée et rayonnement, Québec, Conseil de la famille et de l'enfance, 76.

Dandurand R. (2005). Parentage multiple et partition des responsabilités, in Ouellette F.R., Joyal R., Hurtubise R. (dir.), Familles en mouvance: quels enjeux éthiques?, Québec, PUL-IQRC, «Culture et société», 227-240.

Eme B., Fraisse L. (2005). La gouvernance locale de la diversification des modes d'accueil: un nouvel enjeu de «cohésion sociale», Recherches et Prévisions, n 80, 7-23.

INSEE (2008). Femmes et Hommes - Regards sur la parité, Paris, INSEE, «INSEE Références », 238.

ISQ (2008). État du marché du travail au Québec: le point en 2007, Québec, Institut de la statistique du Québec, coll. «Travail et rémunération», 39.

Jenson J. (2004). Changing the paradigm: Family responsibility or investing in children, The Canadian Journal of Sociology, 29 (2): 169-192.

Lavallée D. (2002). Travailler un peu, beaucoup, comment?, in Tremblay D.G., Dagenais L.F. (dir.), Rupture, segmentations et mutations du marché du travail, Québec, Presses de l'Université du Québec, 117-124.

Lemière S., Marc C. (2006). Qualité des emplois et genre: les effets croisés des statuts et des horaires de travail, Cahiers de la Maison des Sciences économiques, n 74, 29.

Petit P. (2006). Les écarts de salaires horaires entre hommes et femmes en 2002: une évolution possible de la discrimination salariale, Premières Synthèses, no 22.1, 8.

Revillard A. (2006). La conciliation travail-famille: un enjeu complexe pour le féminisme d'État, Recherches et Prévisions, no 85, 17-27.

Statistique Canada (2006). Aperçu sur l'emploi du temps des Canadiens 2005, Ottawa, Statistique Canada, 70.

En ligne: <http://www.statcan.gc.ca/bsolc/francais/bsolc?catno=12F0080XIF\#olcinfopanel>.

Tremblay D.G. (2008a). Conciliation emploi-famille et temps sociaux, Québec/Toulouse, Presses de l'Université du Québec, 342.

Tremblay D.G. (2008b). Vers une articulation des temps sociaux tout au long de la vie; l'aménagement et la réduction du temps de travail, Québec, Presses de l'Université du Québec, (à paraître).

Tremblay D.G. (2008c). Les politiques familiales et l'articulation emploi-famille au Québec et au Canada, in Bigras N., Cantin G. (dir.), Les services de garde éducatifs à la petite enfance du Québec-Recherches, réflexions et pratiques, Québec, Presses de l'Université du Québec, 13-26.

Tremblay D.G. (dir.) (2008d). Flexibilité, sécurité d'emploi et flexicurité: les enjeux et défis, Québec, Presses de l'Université du Québec, 368.

Tremblay D.G. (2004). Économie du travail: Les réalités et les approches théoriques, Montréal, Éditions Saint-Martin et Télé-université, 484.

Tremblay D.G. (2003). Articulation emploi-famille: Comment les pères voient-ils les choses?, Politiques sociales, 63 (3-4): 70-86. 